Indian J Anim Health (2021), 60(2): 172-182

DOI: https://doi.org/10.36062/ijah.2021.04021

\title{
In ovo feeding: Viewpoints on the current status, application and prospect in poultry
}

\author{
K. K. Pandey ${ }^{1}$, S. Koley ${ }^{1 *}$, B. K. Ojha ${ }^{1}$, N. Kurechiya ${ }^{1}$, S. Singh ${ }^{1}$ and A. Singh ${ }^{1}$ \\ ${ }^{1}$ Department of Animal Nutrition, College of Veterinary Science and Animal Husbandry, Nanaji \\ Deshmukh Veterinary Science University, Rewa- 486 001, Madhya Pradesh, India
}

\begin{abstract}
Incubation period including pre-hatch few days are the critical time during which the embryo undergoes rapid growth and development, and prepares for emergence and life outside the egg. This period is also important to chicken survival during the first 1-3 days post-hatch when stored glycogen and residual yolk components aid in the transition to feeding grains. In practical conditions, the time-consuming process in the hatching window additionally hinders newly hatched chicks' access to feed. Therefore, to supply nutrients timely and properly at the very early stage of life of the birds, the concept of in ovo feeding as early nutritional intervention is coming up. This technique is promising to yield numerous advantages, including reduced post-hatch morbidity and mortality, greater efficiency of nutrient utilization at an early age, enhanced immune response to enteric antigens, reduced occurrence of developmental skeletal disorders, improved muscle enlargement and breast meat yield. However, in ovo feeding method needs to be applied in order to supply the embryo with additional nutrients prior to hatching, and those nutrients would likely be utilized by the chick throughout the perinatal period. Further advancement of in ovo feeding system can lead to improved feed conversion efficiency, early attainment of marketable body weight and overall performances of chicks. If the present limitations of in ovo feeding technology are properly addressed, its wide acceptability and implementation are highly prospective in poultry.
\end{abstract}

Key words: Early nutrition, Gut development, In ovo feeding, Postnatal performance, Prenatal

\section{Highlights}

- This article will serve as the valuable source of information of the present status of in ovo feeding in poultry.

- In ovo feeding method with its many positive nutritional responses at the perinatal stage of chicks will draw interest for further research.

- This review will help to study for the development of a standard method or methods of in ovo feeding.

- It will give significant insight of early nutrition with the prospects for nutritionists to increase poultry production to the optimal level.

- It will provide a road map to address the limitations of in ovo feeding technology for its large scale implementation and acceptability as a standard feeding system in poultry.

\section{Introduction}

The poultry production system continues to advance globally. Escalating population, better purchasing capacity and awareness about human health and nutrition have been strong drivers of the incessant growth of poultry production. Such revolution is entailed with considerable investments in several aspects including technical feeding. From 1957 to 2005, the overall live weight of broiler chicken has been amplified by more than $400 \%$. This is achieved now over a shorter period of time. The feed conversion ratio (FCR) has diminished every year on average by $2.55 \%$. Broiler chickens consumed about $2.85 \mathrm{~g}$ of feed for $1 \mathrm{~g}$ of body weight gain in 1957, while the birds ate about $1.67 \mathrm{~g}$ of feed for the same weight gain in 2005 (Zuidhof et al., 2014). Currently,

"Corresponding Author, E mail: skoley2008@gmail.com 
broiler chicken reaches market weight in about 5-6 weeks. The period encompassing the duration of incubation of 21 days plus 10 days post-hatch represents around 50\% of the broiler's lifespan.

Pre-hatch few days are the critical time during which the embryo undergoes rapid growth and development, and prepares for emergence and life outside the egg. This period is also important to chicken survival during the first 1-3 days post-hatch when stored glycogen and residual yolk components aid in the transition to feeding grains (Lilburn, 1998). In market situations, immediately hatched chicks undergo fasting due to unavoidable farming procedures of hatching gaps, sexing, vaccination, and transportation, which can take a significant amount of time, known as hatching window. Therefore, any factor that promotes or delays development, as well as growth in the perinatal (pre-hatch or prenatal and posthatch or postnatal) period, would have obvious consequences on the whole growth and performance of chicks.

Experimental works centred on such time gap denoted the reality that chicks that had late access to feed showed reduced feed conversion efficiency, growth rate, required more days to attain market weight, mortality in chicks, impaired muscle growth and carcass weight (Noy and Sklan, 1999). Early access to feed does the chief task for the development of the gut (Uni et al., 2003; Moran, 2007). The critical thing is that the digestive tract grows faster than any other body system in the earlier life stage of chicks. Deferred feeding adversely influences the function of the gut. Quick availability of feed, as well as water after hatching, showed a noteworthy favourable effect on the live weight of the broiler; however, this is virtually challenging to give feed as well as water in a commercial setup. The timeconsuming process in the hatching window additionally hinders chicks' access to feed. Therefore, to supply nutrients timely and properly at the very early stage of life of the birds for their better development, the concept of in ovo feeding as early nutritional intervention is coming up.

\section{Embryonic development and prenatal nutrition in chick embryo}

Prenatal nutrition is an ongoing research topic. It is not fully understood yet. In poultry, embryo development starts with the fertilization of female gamete with male gamete resulting in zygote formation in the infundibulum. After fertilization, the layer of albumen, shell membrane and eggshell are put surrounding the developing embryo over the yolk. The chick embryo and hatchling rely upon the nutrients deposits in the egg compartments (yolk, albumen and shell). During the 21 days of embryonic development, the chick embryo utilizes essential nutrients from the egg compartment for tissue growth, extraembryonic tissue development and for its energy needs. The content of albumen is approximately 65$75 \%$ of the total content of eggs and consists of $12 \%$ protein and approximately $88 \%$ water all of these two components are completely used by the embryo at the time of incubation (Romanoff, 1960). While yolk is composed of protein ( $15 \%$ of the total yolk), fat (33\%), carbohydrates (less than 1\%) as well as water (about 50\%); yet, such contents bank significantly on the weight of the egg, strain (genetic) plus the age of hen (Vieira and Moran, 1999).

When incubation begins, germ growth resumes. A visible vascular system aids the germ in the absorption of the nutrient from the surrounding albumen (Deeming, 1989) and yolk (Litke and Low, 1975). Oxygen access is limited to simple diffusion at this point (Baumann and Meuer, 1992); therefore, the energy supply is dependent on glycolysis of glucose to lactic acid until the chorioallantoic compartment is formed (Moran, 2007).

Rapid vascular system development enables the exchange of oxygen plus carbon dioxide with shell pores (Tullett, 1984), and fatty acid $\beta$-oxidation becomes the primary energy source 
for embryo development. Around e8 (8 days old embryo), with chorioallantoic respiration established, the vascular system is fully developed to ensure $\mathrm{O}_{2}-\mathrm{CO}_{2}$ exchange (Levinsohn et al., 1984). The embryo is structurally completed by e14, and oxygen consumption reaches a plateau (Moran, 2007).

During the last week of embryo development, albumen enters the amniotic sac, and the amniotic fluid, along with albumen, is orally consumed by the embryo. The albumenamniotic mixture enters the yolk sac through the stalk to permit partial absorption of protein by enterocytes. Gluconeogenesis increases to ensure a sufficient supply of glucose for glycogen accretion in the liver and muscles (Moran, 2007).

Emergence starts when internal pipping occurs around e20. Oxygen supply becomes limited, and energy support is mainly from glucose metabolism provided by the glycogen reserves. The residual yolk sac is internalized and conserved for the transition during the first two days post-hatch. Upon hatch, the liver and intestines continue to develop to prepare for the transition to feed consumption and digestion. During this latter period, the remarkable transformation in physiological system and metabolism occur to ensure the transition of the hatchling. Any interference during this transition period may significantly affect embryo survival or result in suboptimal growth performance after hatch (Moran, 2007).

Overall, embryo development can be divided into three phases. The first week features are the establishment of germ and chorioallantoic compartment formation. Embryo completion, as well as $\mathrm{O}_{2}-\mathrm{CO}_{2}$ exchange of chorioallantoic respiration, occurs in the second week. The last phase includes the accumulation of glycogen in the liver and muscle tissues, commencement of respiration in lungs, internalization of the remaining yolk sac, shell pipping and emergence.

Digestion and absorption of nutrients during incubation take place through various routes: The first route is a visible vascular system that aids the germ in the absorption of nutrients from the surrounding yolk (Litke and Low, 1975) and albumen (Deeming, 1989) in early incubation. Oxygen access is limited to simple diffusion at this point (Baumann and Meuer, 1992); therefore, the energy supply is dependent on glycolysis of glucose to lactic acid until the chorioallantoic compartment is formed (Moran, 2007). The second route is absorption all the way through the membrane of the yolk sac (YSM) directly into the blood. Yolk's particles with 25-30 nm size, comprising principally very-low-density lipoproteins, are engulfed by YSM epithelial cells by endocytosis. The third route is the oral route. From e17, embryos start feeding albumin mix amniotic fluid, which helps in the gastro intestinal tract (GIT) development of the chick embryo. The fourth route of consuming yolk nutrients during incubation is by their transfer directly from the yolk into the intestine through the yolk stalk, probably by peristaltic movements. By this route, the glycogen, fat, protein, vitamins and minerals are subjected to the chick pancreatic enzyme action and can be absorbed by the embryonic intestine and sustain the developing embryo (Moran, 2007; Uni, 2017).

Yadgary et al. (2010) reported that embryos contain the capability to enhance the fat intake from the sac of yolk to make the essential quantity of fat available for the process of hatching. During the final 2- 3 days of hatching, because of inadequate oxygen, fatty acids do not go through oxidation to generate necessary energy. So, needed energy is obtained from glucose's anaerobic type of catabolism from stores of glycogen plus gluconeogenesis of amino acids, glycerol, etc. These findings give an idea that a developing embryo requires the supply of energy from external sources for better development. The concentration of phosphorus $(\mathrm{P})$, iron $(\mathrm{Fe})$ as well as zinc $(\mathrm{Zn})$ was decreased from e0 to e17.5. The level of calcium $(\mathrm{Ca})$, magnesium $(\mathrm{Mg})$ and strontium (Sr) was augmented from e0 to e17.5. The level of copper $(\mathrm{Cu})$, potassium $(\mathrm{K})$ as well as 
sodium (Na) was increased in the beginning (e0 to e6.5) but reduced afterwards. No change occurred in the concentration of manganese $(\mathrm{Mn})$ in the yolk from e0 to e17.5. Substantial altering in the level of yolk minerals takes place at the time of incubation and is apparently linked with mobilization from shell reserves and flux between yolk and albumen (Hopcroft et al., 2018). Micronutrient use by growing embryos depends on albumin, yolk, and shell. As far as the mineral is concerned, the shell is the chief source of $\mathrm{Ca}, \mathrm{Mg}$, and $\mathrm{Sr}$; the albumen supplies the greater part of $\mathrm{K}$ and $\mathrm{Na}$; and the yolk provides $\mathrm{P}, \mathrm{Cu}, \mathrm{Fe}, \mathrm{Mn}$, and $\mathrm{Zn}$ (Yair and Uni, 2011).

Such scientific advancements in embryo development and prenatal nutrition of chicks have made a strong basis for the origin and evolution of in ovo feeding method to its present status and application.

\section{In ovo technology}

One of the useful tools for precision poultry farming and production is the in ovo technology for transforming the situations within the egg through the application of vaccines, nutrients and other bioactives. With this technique, one can deposit a definite quantity of cautiously chosen material into a particular location inside an incubating egg. In ovo technology is targeted for the most decisive time in the chick's development, that is, the perinatal period. The perinatal period extends from the last days of the egg's incubation to the initial few days post-hatching. At this time, the embryo needs to adjust to a change in food, which is from the fat-rich to the carbohydraterich, as well as contact with environmental microbes. In commercial settings, the hatching window is a necessary evil and so unavoidable. Because of these various factors, in ovo method has been developed to facilitate manipulation of the chicken embryo before hatching. In principle, this technology is basically dependent on the delivery of matters by mechanical means straight inside the incubating egg.
First, in ovo inoculation was used for the vaccination purpose against Marek's disease (MD) on the $18^{\text {th }}$ day of incubation and observed better immunization (Sharma and Burmester, 1982). In ovo, immunization is mainly used for MD, Gumboro, Ranikhet and Avian Influenza. However, in ovo technology as a tool of prenatal nutrition is still an area of exploration, and its application is being investigated to deliver carbohydrates, peptides, vitamins, prebiotics, probiotics, synbiotics, etc. Such precise manipulation of the embryo may develop the vigor and resilience of the hatched chicks, which contributes to their further posthatching growth.

\section{In ovo feeding}

In ovo feeding is a technique of providing essential nutrients in the egg itself. Early nutrients access has a fundamental function in the initial stage of growth and utilization of nutrients by chicks. Rapid development, feed utilization efficiency, getting early to marketable bodyweight (Bakyaraj et al., 2012), increased perinatal development (Ferket and Qureshi, 1992) can be achieved with in ovo method of feeding. The supplementation of macro and micronutrients and probiotics also causes a valuable outcome on hatchability as well as disease resistance of hatchlings (Uni and Ferket, 2003).

\section{Different routes of in ovo feeding}

In ovo feeding of nutrients is done via intraembryonic and extraembryonic routes. The intra-embryonic route involves direct inoculation of nutrients into embryonic tissue. On the other hand, in extraembryonic routes, nutrients are inoculated into different extraembryonic contents, viz. yolk sac, albumen, amnion, allantois and chorion and air cell, from where nutrients are absorbed by various mechanisms. According to the time of inoculation, it is defined as early and later stage from e12.

The development of egg-laying birds after hatching is improved through feeding a subject 
before hatching. The subject should be fed with nutrients or an enteric modifier. The nutrient composition preferably should have a minimum of one amino acid, protein, peptide, or carbohydrate. A favourite enteric modulator is hydroxymethyl butyrate. Feeding may be done by giving the nutrients and/or enteric modulator in the amnion where these are orally consumed by the subject (Uni et al., 2003). Therefore, the nutrients can be better supplemented to the developing embryo via the amniotic sac at the later phase of growth, i.e. 18 days of incubation to give a nonstop supply of vital nutrients during the first few days (3- 4 days) of the after-hatch period, so facilitating enteric development and metabolism. This is distinct from other methods of in ovo nutrient administration, for which reason, it was patented with the United States patent US 6592878 B2 (2003) by Uni and Ferket (2003).

The three crucial determinants for in ovo feeding include route of injection, the timing of injection and dose of nutrients (Ohta and Kidd, 2001). Presently 18 to $24 \mathrm{G}$ needles or specialized Inovoject systems are used for injecting the nutrients. After the injection of the egg with the sterile needle under the controlled environment, the site of injection is closed with the paraffin film and the egg is placed into an incubator. The ideal time to administer nutrients in an in ovo feeding programme is 456 hours from fertilization that is roughly e 19 through the amniotic route (Kornasio et al., 2011).

\section{Nutrients for in ovo feeding}

The main purpose of in ovo feeding is to make the embryo able for faster post-hatch growth. Supplying the embryo with exogenous nutrients would allow the GIT to build up the structures and functionality to correctly digest and absorb nutrients instantaneously when an exogenous nutritional supplement is provided after hatch (Uni and Ferket, 2003). These exogenous nutrients, together with the yolk sac reserves, can contribute not only to maintaining the systems and metabolism already established but also to continuing growth, development, and proper nutritional condition (Noy and Skalan, 1998). Feeding of carbohydrates, proteins, and amino acids, vitamins or other modulators through in ovo injection have been evaluated (Bakyaraj et al., 2012; Araújo et al., 2019; Han et al., 2019; Xu et al., 2019). Carbohydrates commonly act as a glucose source and are essential for the process of hatching and hatchling development (Uni et al., 2003). With eggs inoculated with carbohydrates such as, maltose, sucrose and dextrose, it was observed that the extra source of energy improved the development of goblet cells and augmented the surface area of villi in the intestine. Al-Murrani (1982) first experimented with the amino acid supplement to the yolk sac at the 7 day of incubation. Outcomes showed that the embryo did not utilize the amino acids till the late-stage embryonic development to gain. Both Filho et al. (2018) and $\mathrm{Xu}$ et al. (2019) mentioned that the supplementation of amino acids incited the development of GIT and hatchability. Nutrients that can be used in ovo feeding include carbohydrates, all amino acids, fatty acids, vitamins, and other modulators.

\section{In ovo feeding and gut development}

Early access to feed does a key job in the development of the gut (GIT). The critical thing is that the digestive tract grows faster than any other body system in the earlier life stage of chicks. The method of in ovo feeding permits the deliverance of a range of supplements straight into chicken embryos; facilitate the early founding of a healthy gut microbiome prior to it is exposed to any pathogenic bacteria.

In the final phase of incubation, nutrients, which are ingested with the amniotic fluid, arrive at the intestine of the embryo (Romanoff, 1960; Moran, 2007). Through this time, the intestine of the embryo experiences molecular, cellular and morphological changes, rapid proliferation and differentiation of enterocytes, enhanced capacity of absorption and nutrient uptake, and higher gene expression concerned in the process of digestion and absorption in the 
epithelial enterocytes (Geyra et al., 2001; Uni et al., 2003). mRNA of the digestive enzymes sucrase-isomaltase (SI) and aminopeptidase $\mathrm{N}$ (APN) is abundant in the intestine of chick embryo on e19 (Uni et al., 2003). Those of peptide transporter (PepT1), as well as sodium-glucose transporter (SGLT1), enhance significantly from e18 to e21 (Chen et al., 2005; Gilbert et al., 2007), and coding of genes for various transporters of amino acids demonstrate elevated levels of expression on e18 (Filho et al., 2018). Nutrients of in ovo feed supplied by in ovo feeding are then exposed to the tissues of the gut after the embryo orally ingests the amniotic contents before the emergence of air cells (Moran, 2007). This also makes the nutritional condition of the hatchlings better by means of speeding up the development of the gut for superior competence of digestion and nutrient absorption.

\section{In ovo feeding and immune response}

In ovo feeding may also stimulate immunocompetence during early stages by enhancing the protective function of enteric mucosa. Hatchlings are highly vulnerable to the colonization of gut pathogens because of the nominal competitive exclusion by symbiotic microflora, which colonizes the mucin layer of the mucosa of the gut. The primary defence against gut infection is the mucus gel layer of the intestinal epithelium. Kidd (2004) suggested that the nutrients (fat, amino acids or watersoluble vitamins and trace metals) use at a quantity higher than the requirements confers immune benefits to poultry. Providing natural nutrients by in ovo feeding maintains and hastens the development of the gut, giving the bird a perfect beginning point towards enhanced immune response to enteric antigens. Uni et al. (2003) noticed that in ovo feeding augmented surface area of villus at hatch and 3 day posthatch by approximately $27 \%$ and $21 \%$, respectively. Besides, the fraction of goblet cells containing acidic mucin was enhanced by $50 \%$ over controls at $36 \mathrm{~h}$ after in ovo feeding, which was related to increase mucin mRNA expression. Therefore, in ovo feeding perhaps will be helpful for improving the colonization resistance of enteric pathogenic organisms of newborn and young chicks.

Bhanja and Mandal (2005) reported that in ovo injection of vitamin E (0.25-0.50 IU) may be sufficient to augment post-hatch development as well as humoral immune response; while linoleic acid may enhance cellmediated immunity in broiler chickens and increases lipid in brain tissue as well as the status of antioxidants in hatched chicks. Researchers have definitely recognized that in ovo feeding is helpful for early immune response as well as growth. Greater enlightenment of the needs of main immune-modulatory nutrients will be helpful for the nutritionists to formulate in ovo feeds to improve immunity and the overall health of birds. The aptness of products for in ovo application in poultry nutrition and production necessitates further investigation.

\section{In ovo stimulation and embryonic microbiome}

The concept of in ovo stimulation has come from that of perinatal programming. The concept of perinatal programming was originated from human studies, which gave the hypothesis that a stimulus (or adverse factor) given to a foetus during the early stage of its development could lead to permanent phenotypic alteration (Calkins and Devaskar, 2011).

The key notion of the in ovo stimulation is centered on stimulating the colonization of the embryonic gastrointestinal tract with its native microbiota that will help in establishing the optimal microbiota already existing during the incubation of the egg. It is shown that delivering $0.2 \mathrm{~mL}$ volume of dissolved bioactive stimulus right on day 12 of incubation of egg can kick off an entire flow of incidents on various phenotypic concentrations, from modulation of gene expression to growth performance. Scientists, K. Gulewicz and M. Bednarczyk, have developed and patented this procedure (Siwek et al., 2018). 
The majority of the microbes are used as probiotics in animal feeding and belonging to various species of bacteria like Lactobacillus, Bacillus, Bifidobacterium and Enterococcus, yeasts like Saccharomyces cerevisae and Saccharomycces bourlardii as well as fungi like Aspergillus oryzae and Candida pintolepsii can also be meant for the same rationale.

In ovo stimulation is especially significant in the industrial production of chicken with a poor microbial profile leading to enteric microbial infestation and infectious disease outbreaks, which become even more customary after the removal of growth-promoting antibiotics from various global markets.

\section{In ovo feeding and postnatal performances of chicks}

Postnatal performances of chicks subjected to in ovo feeding are shown in Table 1.

Table 1. In ovo feeding and postnatal performances of chicks

\begin{tabular}{lcccc}
\hline Nutrient injected & $\begin{array}{c}\text { Day of } \\
\text { injection }\end{array}$ & $\begin{array}{c}\text { Route of } \\
\text { injection }\end{array}$ & Effects on performances & Reference \\
\hline
\end{tabular}

\begin{tabular}{lllll}
$\begin{array}{l}\text { Dextrin }(10 \%), \beta- \\
\text { hydroxy- } \beta-\end{array}$ & Amniotic \\
$\begin{array}{l}\text { methylbutyrate-calcium } \\
(0.4 \%) \text { salt }\end{array}$ & $18^{\text {th }}$ day & $\begin{array}{l}\text {-Elevated hepatic glycogen } \\
\text { contents of chick }\end{array}$ & $\begin{array}{l}\text { Kornasio } \\
\text {-Increased muscle growth and } \\
\text { bodyweight }\end{array}$ & et al., 2011 \\
\hline
\end{tabular}

Probiotics

(Enterococcus faecium

$5 \times 10^{9} \mathrm{cfu} / \mathrm{egg}$ and

$17.5^{\text {th }}$ day $\begin{aligned} & \text { Amniotic } \\ & \text { sac }\end{aligned}$

-Decrease in the number of

de Oliveira

Salmonella enteritidis in chicks

et al., 2014

Bacillus subtilis $5 \times$

$10^{12} \mathrm{cfu} / \mathrm{egg}$ )

Nano zinc (Zn) (20, 40,

60 and $80 \mu \mathrm{g} / \mathrm{egg})$

Nano copper $(\mathrm{Cu})(4$,

$8,12$ and $16 \mu \mathrm{g} / \mathrm{egg})$

Nano Se $(0.075,0.15$,

0.225 and $0.3 \mu \mathrm{g} / \mathrm{egg}$ )

$\begin{array}{llll}18^{\text {th }} \text { day Amniotic } & \text {-Best feed efficiency at } \mathrm{Zn}-40 & \text { Joshua et al., } \\ \text { sac } & \mu \mathrm{g}, \mathrm{Cu}-4 \mu \mathrm{g} \text { and, Se }-0.225 & 2016 \\ & \mu \mathrm{g} / \mathrm{egg} \\ & \text {-Highest breast muscle } \\ & \text { percentage at } \mathrm{Cu}-12 \mu \mathrm{g} / \mathrm{egg}\end{array}$

-Improved the chicks' oxidative

Vit. E $\left(0.0,27.5,38.5, \quad 18^{\text {th }}\right.$ day Amniotic state, which led to

49.5 and $60.4 \mathrm{IU})$

$\mathrm{Sac}$

improvements in incubation

Araújo et al., results, chick quality and 2019

performance results

Threonine $(17.5,35$,

52.5 and $70 \mathrm{mg}$ threonine/mL)

$18^{\text {th }}$ day $\begin{aligned} & \text { Amniotic } \\ & \text { sac }\end{aligned}$

-Beneficially affected the morphological and functional development of the intestinal

Filho et al., mucosa, which ensured improved performance of chicks at hatch and at post hatch 21 day period. 
Cont. Table 1.

\begin{tabular}{|c|c|c|c|c|}
\hline Nutrient injected & $\begin{array}{l}\text { Day of } \\
\text { injection }\end{array}$ & $\begin{array}{l}\text { Route of } \\
\text { injection }\end{array}$ & Effects on performances & Reference \\
\hline $\begin{array}{l}\text { L-ascorbic acid }(3,6, \\
\text { and } 12 \mathrm{mg})\end{array}$ & $17^{\text {th }}$ day & $\begin{array}{l}\text { Amniotic } \\
\text { sac }\end{array}$ & $\begin{array}{l}\text {-Lasting positive effects on the } \\
\text { post hatch growth, leg muscle } \\
\text { development, and systemic } \\
\text { antioxidant capacity of broilers } \\
\text { at } 42 \mathrm{~d}\end{array}$ & $\begin{array}{l}\text { Zhang } \\
\text { et al., } 2019\end{array}$ \\
\hline $\begin{array}{l}\text { L-leucine } 34.5 \mu \text { mol } \\
\text { L-Leucine dissolved in } \\
500 \mu \mathrm{L} \text { sterile water/egg }\end{array}$ & $\begin{array}{l}14^{\text {th }} \text { and } \\
19^{\text {th }} \text { day }\end{array}$ & $\begin{array}{l}\text { Amniotic } \\
\text { sac }\end{array}$ & $\begin{array}{l}\text {-Thermo tolerance in broilers } \\
\text { increased }\end{array}$ & $\begin{array}{l}\text { Han et al., } \\
2019\end{array}$ \\
\hline $\begin{array}{l}\text { Methionine ( } 20 \text { and } 30 \\
\mathrm{mg}) \text { and Lysine } \\
(20 \text { and } 30 \mathrm{mg})\end{array}$ & - & Egg & $\begin{array}{l}-90^{\circ} \text { angle of the needle used for } \\
\text { nutrient inoculation was safer for } \\
\text { the embryos-Improved intestinal } \\
\text { morphometry }\end{array}$ & $\begin{array}{l}\text { Groff- } \\
\text { Urayama } \\
\text { et } a l ., 2019\end{array}$ \\
\hline
\end{tabular}

-Lower hatching rates and a longer hatching -Higher percentage of chicks with poor physical quality score (<71.0 points) obtained among the chicks from eggs injected at the rate of $0.0,0.35$, $0.45,0.55$, and 0.65 $\mathrm{mg} / 0.5 \mathrm{~mL}$ of sterilized in ovo injected using a manual needle in broiler eggs.
Canthaxanthin (CCX) and distilled water were

\section{$17.5^{\text {th }}$ day}

Amniotic cavity with 0.55 and $0.65 \mathrm{mg}$ of CCX -Higher total proteins and catalase activity in the livers of the chicks injected with CCX -Broiler chicks in the control group (0.0 mg of CCX) presented higher body weight and body weight gain during 1 to 7 and 7 to 14 day of afterhatch.

-The viability (\%) of chicks at 1 to 14 day of afterhatch decreased with inoculation greater than $0.45 \mathrm{mg}$ of CCX in ovo -Although the CCX showed an improvement in oxidation status of chicks, the hatchability and performance of broilers decreased
Araújo

et al., 2020
-Increase in the body weight and breast meat yield of broilers

Fatemi

-Decrease in the circulating et al., 2021 $\mu \mathrm{g} 25$ - hydroxyvitamin D3 (OHD3), $2.4 \mu \mathrm{g}$ D3 $+2.4 \mu \mathrm{g} 25 \mathrm{OHD} 3$. $18^{\text {th }}$ day Egg plasma $\alpha$-1-acid glycoprotein (AGP) concentrations of broilers 


\section{Future studies}

There are still many limitations of in ovo feeding. To remove these limitations following problems are required to be solved through further studies:

1. Needs of appropriate nutritional products for in ovo administration

2. Needs of standardization of nutrients composition, doses of nutrients, concentration and viscosity of nutrient solutions, volumes of solutions, timing during pre-hatch period, routes, sites of injection, type of needles, sealing of holes, etc. to obtain different or all desirable post-hatch effects

3. Needs to remove doubt in a decrease in hatchability as a result of in ovo feeding of some nutrients

\section{Conclusion}

Although in ovo feeding method has produced many positive nutritional responses at the perinatal stage of chicks, such advantages, whether this method is sustainable for growth and production in poultry is still not well established. There are still lots of debates among scientists on the long-term benefits of in ovo feeding. It is obvious that there are inconsistent outcomes of research trials on in ovo feeding. So, this is necessary to develop a standard

\section{REFERENCES}

Al-Murrani W, 1982. Effect of injecting amino acids into the egg on embryonic and subsequent growth in the domestic fowl. Br Poult Sci, 23(2): 171-174, doi: $10.1080 / 00071688208447943$

Araújo ICS, Café MB, Noleto RA, Martins JMS, Ulhoa CJ et al., 2019. Effect of vitamin E in ovo feeding to broiler embryos on hatchability, chick quality, oxidative state, and performance. Poult Sci, 98(9): 3652-3661, doi: 10.3382/ps/pey439

Araújo, ICS, Café MB, Mesquita MA, Caiado BN, Faria AM et al., 2020. Effect of a commercial product containing canthaxanthin for in ovo feeding to broiler embryos on hatchability, chick quality, oxidation status, and performance. Poult Sci, 99(11): 5598-5606, doi: 10.1016/j.psj.2020.08.044 method or methods of in ovo feeding. This technique is promising to yield numerous advantages, including reduced post-hatch morbidity and mortality, greater efficiency of nutrient utilization at an early age, enhanced immune response to enteric antigens, reduced occurrence of developmental skeletal disorders, improved muscle enlargement and breast meat yield. It has set up a novel opportunity for early nutrition that provides prospects for nutritionists to increase poultry production to the optimal level. Many potential nutrients have been tested in this way, and new findings are being reported. If the present limitations of in ovo feeding technology are properly studied and rectified, its large scale implementation and acceptability as a standard feeding system in poultry are highly prospective.

Conflict of interest: Authors have no conflict of interest in this study.

Author's contribution: KKP: The acquisition of data and critically revising the manuscript; SK: Substantial contribution to the conception and design, the acquisition of data and critically revising the manuscript; BKO: Critically revising the manuscript; NK: Substantial contribution to the conception and design; SS: Critically revising the manuscript; AS: Critically revising the manuscript.

Bakyaraj S, Bhanja SK, Majumdar S and Dash B, 2012. Modulation of post-hatch growth and immunity through in ovo supplemented nutrients in broiler chickens. J Sci Food Agric, 92(2): 313-320, doi: 10.1002/jsfa.4577

Baumann R and Meuer HJ, 1992. Blood oxygen transport in the early avian embryo. J Physiol, 72(4): 941-965, doi: 10.1152/physrev.1992.72.4.941

Bhanja SK and Mandal AB, 2005. Effect of in ovo injection of critical amino acids on pre- and posthatch growth, immunocompetence, and development of digestive organs in broiler chickens. Asian-Australas J Anim Sc, 18(4): 524-531, doi: 1 0.5713/ajas.2005.524

Calkins K and Devaskar SU, 2011. Fetal origins of adult 
disease. Curr Probl Pediatr Adolesc Health Care, 41(6): 158-176, doi: 10.1016/j.cppeds.2011.01.001

Chen H, Pan Y, Wong EA and Webb Jr KE, 2005. Dietary protein level and stage of development affect expression of an intestinal peptide transporter (cPepT1) in chickens. J Nutr, 135(2): 193-198, doi: 10.1093/jn/135.2.193

de Oliveira JE, van der Hoeven-Hangoor E, van de Linde IB, Montijn RC and van der Vossen JM, 2014. In ovo inoculation of chicken embryos with probiotic bacteria and its effect on posthatch Salmonella susceptibility. Poult Sci, 93(4): 818-29, doi: 10.3382/ ps.2013-03409

Deeming DC, 1989. Importance of sub-embryonic fluid and albumen in the embryo's response to turning of the egg during incubation. Br Poult Sci, 30(3): 591-606, doi: 10.1080/00071668908417182

Fatemi SA, Alqhtani AH, Elliott KEC, Bello A, Levy AW et al., 2021. Improvement in the performance and inflammatory reaction of Ross 708 broilers in response to the in ovo injection of 25-hydroxy vitamin $\mathrm{D}_{3}$. Poult Sci, 100(1): 138-146, doi: 10.1016/ j.psj.2020.10.010

Ferket PR and Qureshi MA, 1992. Performance and immunity of heat-stressed broilers fed vitamin and electrolyte-supplemented drinking water. Poult Sci, 71(1): 88-97, doi: 10.3382/ps.0710088

Filho A, Ferket PR, Malheiros RD, Oliveira C, Aristimunha PC et al., 2018. Enrichment of the amnion with threonine in chicken embryos affects the small intestine development, ileal gene expression and performance of broilers between 1 and 21 days of age. Poult Sci, 98(3): 1363-1370, doi: $10.3382 / \mathrm{ps} / \mathrm{pey} 461$

Geyra A, Uni Z and Sklan D, 2001. Enterocyte dynamics and mucosal development in the posthatch chick. Poult Sci, 80(6): 776-782, doi: /10.1093/ps/80.6.776

Gilbert ER, Li H, Emmerson DA, Webb Jr KE and Wong EA, 2007. Developmental regulation of nutrient transporter and enzyme mRNA abundance in the small intestine of broilers. Poult Sci, 86(8): 17391753, doi: 10.1093/ps/86.8.1739

Groff-Urayama P, Padilha J, Einsfeld S, Pertile S, Gorges $\mathrm{M}$ et al., 2019. Performance, intestinal morphometry and incubation parameters of broiler chickens submitted to in ovo feeding with different techniques and amino acids. Can J Anim Sci, 99(4): 732-740, doi: 10.1139/cjas-2018-0131

Han G, Yang H, Wang Y, Zhang R, Tashiro Ket al., 2019. Effects of in ovo feeding of L-leucine on amino acids metabolism and heat-shock protein-70, and $-90 \mathrm{~m}$ RNA expressions in heat-exposed chicks. Poult Sci, 98(3): 1243-1253, doi: 10.3382/ps/ pey444

Hopcroft RL, Cowieson AJ, Muir WI and Groves PJ, 2018. Changes to mineral levels in the yolk of meat chicken embryos during incubation. Poult Sci, 98(3): 1511-1516, doi: 10.3382/ps/pey423

Joshua P, Valli C and Balakrishnan V, 2016. Effect of in ovo supplementation of nano forms of zinc, copper, and selenium on post-hatch performance of broiler chicken. Vet world, 9(3): 287-294, doi: 10.14202/vetworld.2016.287-294

Kidd MT, 2004. Nutritional modulation of immune function in broilers. Poult Sci, 83(4): 650-657, doi: 1 0.1093/ps/83.4.650

Kornasio R, Halevy O, Kedar O and Uni Z, 2011. Effect of in ovo feeding and its interaction with timing of first feed on glycogen reserves, muscle growth, and body weight. Poult Sci, 90(7): 1467-1477, doi: 10.3382/ps.2010-01080

Levinsohn EM, Packard Jr DS, West EM and Hootnick DR, 1984. Arterial anatomy of chicken embryo and hatchling. Am J Anat, 169(4): 377-405, doi: 10.1002/ aja.1001690403

Lilburn MS, 1998. Practical aspects of early nutrition for poultry. J Appl Poult Res, 7(4): 420-424, doi: 10.1093/japr/7.4.420

Litke LL and Low FN, 1975. Scanning electron microscopy of yolk absorption in early chick embryos. Am J Anat, 142(4): 527-531, doi: 10.1002/ aja. 1001420410

Moran ET Jr, 2007. Nutrition of the developing embryo and hatchling, Poult Sci, 86(5): 1043-1049, doi: $10.1093 / \mathrm{ps} / 86.5 .1043$

Noy Y and Sklan D, 1998. Metabolic responses to early nutrition. J Appl Poult Res, 7(4): 437-51, doi: 10.1093/ japr/7.4.437

Noy Y and Sklan D, 1999. Energy utilization in newly hatched chicks. Poult Sci, 78(12): 1750-1756, doi: $10.1093 / \mathrm{ps} / 78.12 .1750$

Ohta Y and Kidd MT, 2001. Optimum site for in ovo amino acid injection in broiler breeder eggs. Poult Sci, 80: 1425-1429, doi: 10.1093/ps/80.10.1425

Romanoff AL, 1960. The avian embryo: structural and functional development. Macmillan, New York, USA, pp 1305, doi: 10.1126/science.131.3408.1219-a

Sharma J and Burmester B, 1982. The resistance of Marek's disease at hatching in chickens vaccinated as embryos with the turkey herpes virus. Avian Dis, 26(1): 134-149

Siwek M, Slawinska A, Stadnicka K, Bogucka K, Dunislawska A et al., 2018. Prebiotics and synbiotics - in ovo delivery for improved lifespan 
condition in chicken. BMC Vet Res, 14(1): 402, doi: 10.1186/s12917-018-1738-z

Tullett SG, 1984. The porosity of avian egg shells. Comp Biochem Physiol Part A: Physiol, 78(1): 5-13, doi: 10.1016/0300-9629(84)90083-5

Uni Z and Ferket P, 2003. Enhancement of Development of Oviparous Species by In Ovo Feeding. United States patent US 6592878 B2 (2003). Retrieved from https://repository.lib.ncsu.edu/bitstream/handle/ 1840.2/925/US_6592878_B2_I.pdf?sequence= landisAllowed $=y$ [ [2 $2^{\text {nd }}$ May, 2021]

Uni Z, Smirnov A and Sklan D, 2003. Pre-and posthatch development of goblet cells in the broiler small intestine: effect of delayed access to feed. Poult Sci, 82(2): 320-327, doi: 10.1093/ps/82.2.320

Uni Z, 2017. The effects of in-ovo feeding. Retrieved from https://zootecnicainternational.com/featured/ effects-ovo-feeding/ 2017 [2 ${ }^{\text {nd }}$ May, 2021]

Vieira S and Moran Jr E, 1999. Effects of the egg of origin and chick post-hatch nutrition on broiler live performance and meat yields. Worlds Poult Sci J, 55(2): 125-142, doi: 10.1079/WPS19990009

Xu QQ, Zhang XY, Zou XT and Dong XY, 2019. Effects of in ovo injection of L-histidine on hatch performance and post-hatch development in domestic pigeons (Columba livia). Poult Sci, 98(8): 3194-3203, doi: 10.3382/ps/pez046

Yadgary L, Cahaner A, Kedar O and Uni Z, 2010. Yolk sac nutrient composition and fat uptake in lateterm embryos in eggs from young and old broiler breeder hens. Poult Sci, 89(11): 2441-2452, doi: 10.3382/ps.2010-00681

Yair R and Uni Z, 2011. Content and uptake of minerals in the yolk of broiler embryos during incubation and effect of nutrient enrichment. Poult Sci, 90(7): 1523-1531, doi: 10.3382/ps.2010-01283

Zhang H, Elliott KEC, Durojaye OA, Fatemi SA, Schilling MW et al., 2019. Effects of in ovo injection of Lascorbic acid on growth performance, carcass composition, plasma antioxidant capacity, and meat quality in broiler chickens. Poult Sci, 98(9): 36173625, doi: $10.3382 / \mathrm{ps} /$ pez173

Zuidhof MJ, Schneider BL, Carney VL, Korver DR and Robinson FE, 2014. Growth, efficiency, and yield of commercial broilers from 1957, 1978, and 2005. Poult Sci, 93(12): 2970-2982, doi: 10.3382/ps.2014-04291

Received - 08.05.2021, Accepted - 25.10.2021, Published - 01.12.2021

Section Editor: Dr. A. K. Patra, Associate Editor 